\title{
Teratoma oral: la importancia del diagnóstico prenatal y el trabajo interdisciplinario: un caso clínico
} Epignathus, the importance of prenatal diagnosis and interdisciplinary work: A case report

\author{
Dra. Camila Parellada ${ }^{a}$ Dra. Mónica N. Pose ${ }^{a}$,Dra. Mariana Vázquez Durand ${ }^{a}$ Dra. Yamile Rubies ${ }^{a}$, \\ Dra. Delcys Albas Maubett ${ }^{a}$ Dr. Daniel Saint Genez $z^{a}$ y Dr. Santiago Vidaurreta ${ }^{a}$
}

\section{RESUMEN}

Gracias al avance de la tecnología, es posible realizar el diagnóstico prenatal de distintas malformaciones congénitas que ponen en riesgo la vida del recién nacido. Entre estas, el teratoma oral o epignathus es una forma poco frecuente de teratoma congénito entre los que se localizan en cabeza y cuello. Suelen ser benignosy abarcan el $4 \%$ delos teratomas neonatales. A partir del desarrollo de la técnica de tratamiento intraparto extraútero (EXIT, por su sigla en inglés), que se implementó en los años 90 para mantener la circulación fetal hasta asegurar la vía aérea del recién nacido, se logra planificar una estrategia de manejo multidisciplinario que permite el abordaje correcto de estas patologías.

Se presenta un caso de teratoma oral gigante en una paciente de sexo femenino de 35 semanas de gestación, en quien se aplicó la técnica EXIT, y su evolución posterior.

Palabras clave: teratoma oral, epignathus, EXIT.

\begin{abstract}
Thanks to technological advances, it has been possible to carry out the prenatal diagnosis of different life-threatening congenital malformations. Among these, oral teratoma, or epignathus, is a rare form of congenital teratoma within those located in the head and neck. They are generally benign and comprise $4 \%$ of neonatal teratomas.

From the development of the EXIT technique (ex utero intrapartum treatment), which has been implemented since the 90 's to support fetal circulation until the newborn's airway is secured, it is possible to plan a multidisciplinary management strategy that enables the correct approach of these pathologies. We present a case of giant epignathus in a 35-week gestation female patient, whose airway was secured using the EXIT technique, and follow up.

Key words: oral teratoma, epignathus, EXIT.
\end{abstract}

http:/ / dx.doi.org/10.5546/ aap.2021.e499

a. Sección Neonatología y Departamento de Pediatría, Hospital Universitario CEMIC, Ciudad Autónoma de Buenos Aires, Argentina.

Correspondencia:

Dra. Camila Parellada: camilaparellada@gmail.com

Financiamiento: Ninguno.

Conflictos de interés: Ninguno que declarar.

Recibido: $21-10-2020$

Aceptado: 15-4-2021
Cómo citar: Parellada C, Pose MN, Vázquez Durán M, Rubies Y, et al. Teratoma oral: la importancia del diagnóstico prenatal y el trabajo interdisciplinario: un caso clínico. Arch Argent Pediatr 2021;119(5):e499-e503.

\section{INTRODUCCIÓN}

Los teratomas congénitos se presentan en $1 / 4000$ recién nacidos vivos. Entre estos tumores, el teratoma oral o epignathus constituye el 2 a $4 \%$ (1/35 000-1/200 000), y se presenta con mayor frecuencia en pacientes de sexo femenino, con una relación 3:1. ${ }^{1}$ El tumor puede causar obstrucción grave de la vía aérea y se asocia con mortalidad neonatal elevada ( 80 a $100 \%)^{2}$ El diagnóstico prenatal se realiza, en general, mediante ecografía durante el segundo o tercer trimestre de embarazo, ${ }^{3}$ y provee una oportunidad al equipo tratante de planificar un enfoque terapéutico multidisciplinario intraparto para asegurar la vía aérea y disminuir el riesgo de mortalidad perinatal. ${ }^{4}$ A continuación, se notifica un caso de epignathus gigante tratado de manera satisfactoria mediante la técnica de EXIT a las 35 semanas de gestación.

\section{CASO CLÍNICO}

Llega al servicio de obstetricia de alto riesgo una paciente de sexo femenino de 35 años embarazada, con diagnóstico de hipotiroidismo en tratamiento con levotiroxina. Es secundigesta, con cesárea previa sin antecedentes patológicos de relevancia.

A las 21 semanas de gestación se diagnostica, por ecografía prenatal, un tumor orofacial como probable teratoma de lengua en el feto. Se observa también polihidramnios que requirió amniorreducción en la semana 27. Se mantuvo conducta expectante, con evaluación de la extensión tumoral y seguimiento con resonancia magnética nuclear (RMN) en dos oportunidades hasta la semana 34,3 de edad gestacional, cuando se realiza maduración pulmonar fetal con corticoides, despegamiento de amnios y se 
programa EXIT para la semana 35 de gestación.

Se realiza cesárea con anestesia general, con rotura artificial de membranas y líquido amniótico claro. Manteniendo la circulación placentaria, se recibe una recién nacida de sexo femenino, de 35 semanas de gestación y $2575 \mathrm{~g}$ de peso. Se coloca tubo endotraqueal mediante endoscopia flexible y se traslada a la unidad de cuidados intensivos neonatales (UCIN) para el control evolutivo y posterior evaluación diagnóstica de la extensión (Figura 1).

Se coloca a la niña en asistencia respiratoria mecánica (ARM) y se canalizan los vasos umbilicales. Al examen físico, se constata fisura del paladar blando, labio leporino y masa tumoral que parece originarse en la lengua y se exterioriza por el orificio de la boca y la

FiguRA 1. Nacimiento por cesárea

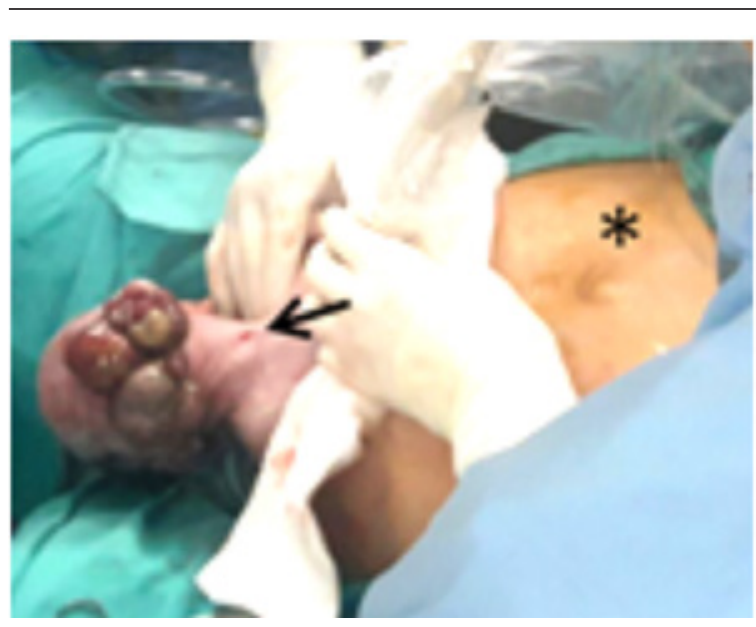

Se observa abdomen materno ${ }^{*}$ ) con incisión de cesárea por donde se extrae al recién nacido (flecha). narina derecha (Figura 2). Se coloca una cubierta plástica estéril que envuelve al tumor, se inicia nutrición parenteral (NPT) y se solicitan estudios complementarios de laboratorio (subunidad $\beta, \propto$-fetoproteína y cariotipo), de diagnóstico por imagen (ecografía cerebral, abdominal y angiotomografía de cabeza, cuello y tórax) y valoración cardiológica con ecocardiograma Doppler color.

Figura 2. Aspecto posnatal inmediato

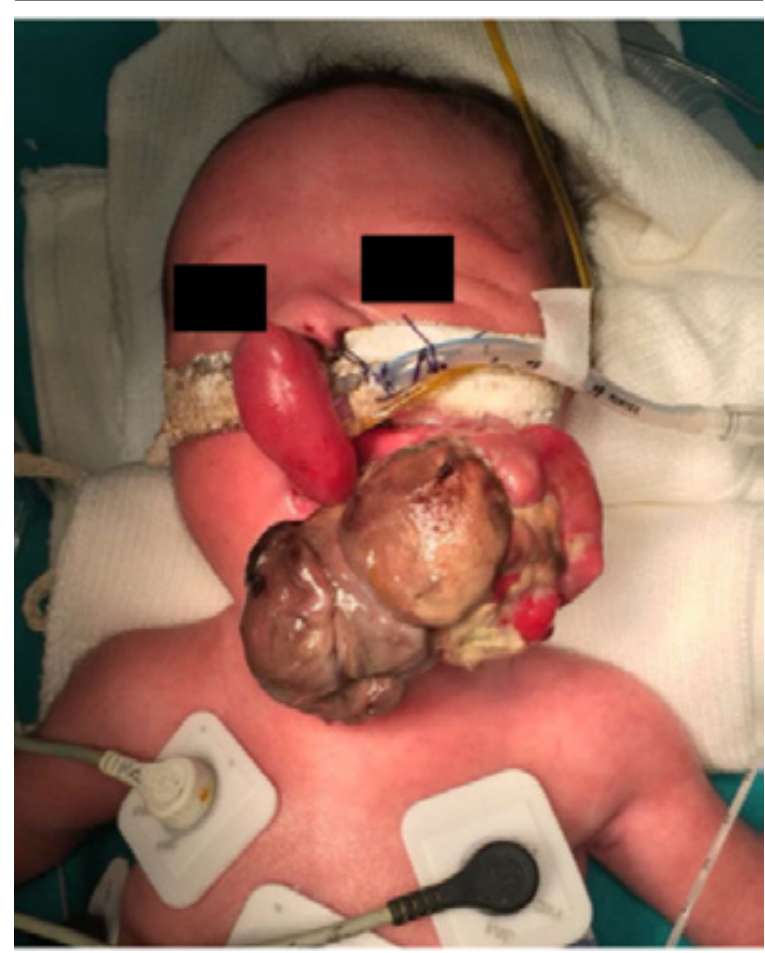

Masa tumoral que parece originarse en la lengua y sale al exterior por el orificio de la boca y la narina derecha.

Figura 3. Angiotomografía
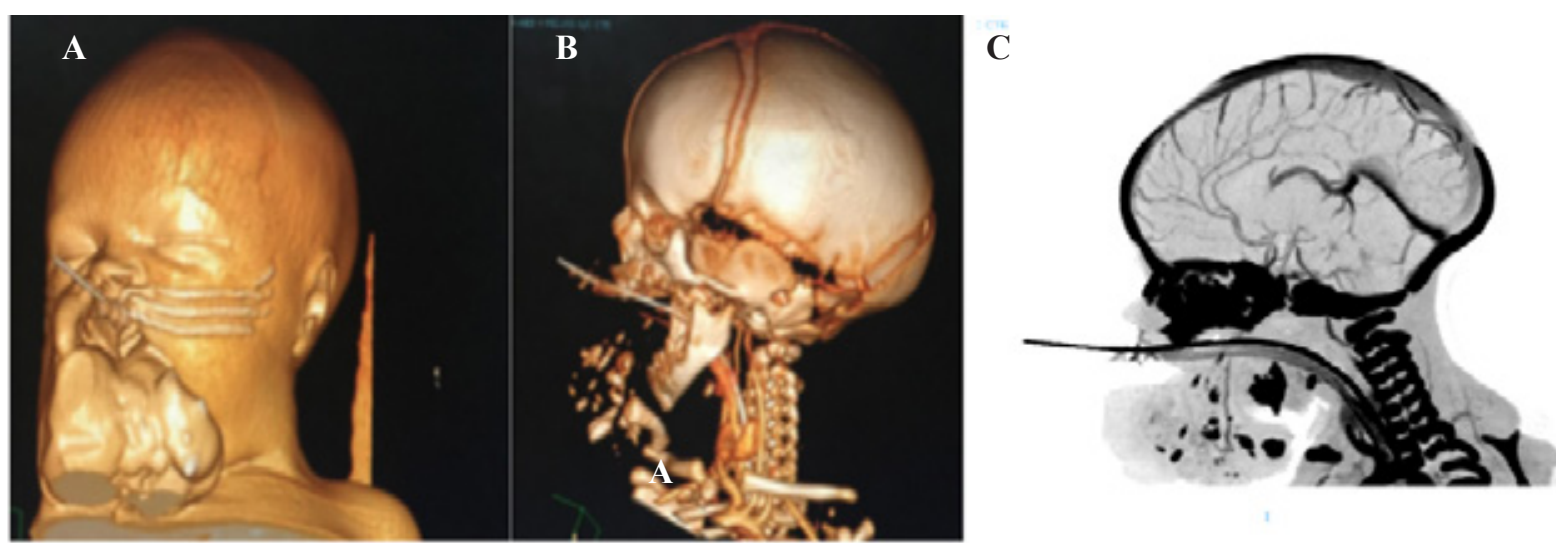

A-C: angiotomografía con reconstrucción 3D de cabeza y cuello. 
La angiotomografía muestra una masa voluminosa heterogénea con áreas cálcicas en su interior y leve realce al administrar contraste, de $65 \times 60 \mathrm{~mm}$ que compromete el paladar duro, el tabique nasal y la lengua, que impresiona depender de la fosa nasal derecha y el piso de la boca (Figuras 3a, 3b y 3c).

A los 5 días de vida, se efectúa la resección del tumor en quirófano y bajo anestesia general. Durante el procedimiento se observa tumor de origen palatino, fisurado, que compromete el pilar anterior izquierdo e invade la fosa nasal derecha, y se realiza la resección. El resto del tumor se encuentra adherido al pilar anterior izquierdo y se realiza la resección completa, con liberación de la vía aérea obstruida (Figura 4a).

Se envían muestras a anatomía patológica de las diferentes regiones de la tumoración, más dos fragmentos de la región nasal, uno del paladar blando y varios rotulados como pilar posterior izquierdo En la masa de la región orofaríngea, se observa, a nivel macroscópico, una formación nodular lobulada de consistencia blanda y color parduzco, se reconocen áreas quísticas y sectores sólidos de consistencia cálcica, mixoide y otros sectores de color pardo-rojizos de aspecto hemorrágico.

A nivel microscópico, el material resecado de la región orofaríngea y nasal presentan revestimiento mucoso y, en la muestra de la fosa nasal, epitelio escamoso con anexos cutáneos y tejidos maduros correspondientes a teratoma, que incluyen mucosa intestinal, cartílago maduro, quistes con epitelio de tipo respiratorio, tejido muscular liso, tejido glial maduro, microcalcificaciones y áreas con pigmento melánico. En parte de la superficie de las muestras se observa erosión de la mucosa de revestimiento y necrosis, recubierta de material fibrino-hemático. En la resección de la lesión nasal se observa, además, tejido pancreático, tanto acinar como islotes de Langerhans.

Durante la internación, la niña presentó enfermedad de membrana hialina y requirió 2 dosis de surfactante. Permaneció con ARM durante 8 días y con cánula nasal por 4 días, luego aire ambiente con buena tolerancia.

Recibió NPT por 17 días, luego comenzó con nutrición enteral por sonda nasogástrica y, debido a que no se registró pasaje de leche a narina y presentó buena coordinación deglutoria evaluada por videodeglución, comenzó a alimentarse por succión con tetina especial para paladar hendido, con buena tolerancia.

Se realizaron determinaciones inmunohistoquímicas. Se detectó citoqueratina AE1-AE3 en los epitelios maduros descritos y cromogranina A en los islotes pancreáticos. La fosfatasa alcalina placentaria y CD117 fueron negativos, OCT4 resultó positivo focal. El material enviado aparte, como el proveniente del paladar blando y del pilar posterior, muestran mucosa sin compromiso por la neoplasia. Esto permite confirmar el diagnóstico de teratoma maduro basicraneal (epignathus).

Al mes de vida se otorga el egreso hospitalario, con controles ambulatorios con pediatra de cabecera, fonoaudiología y odontología.

Cumplido un año de vida, la paciente no presenta evidencia de tumor, y está pendiente la corrección del paladar hendido (Figura 4b).
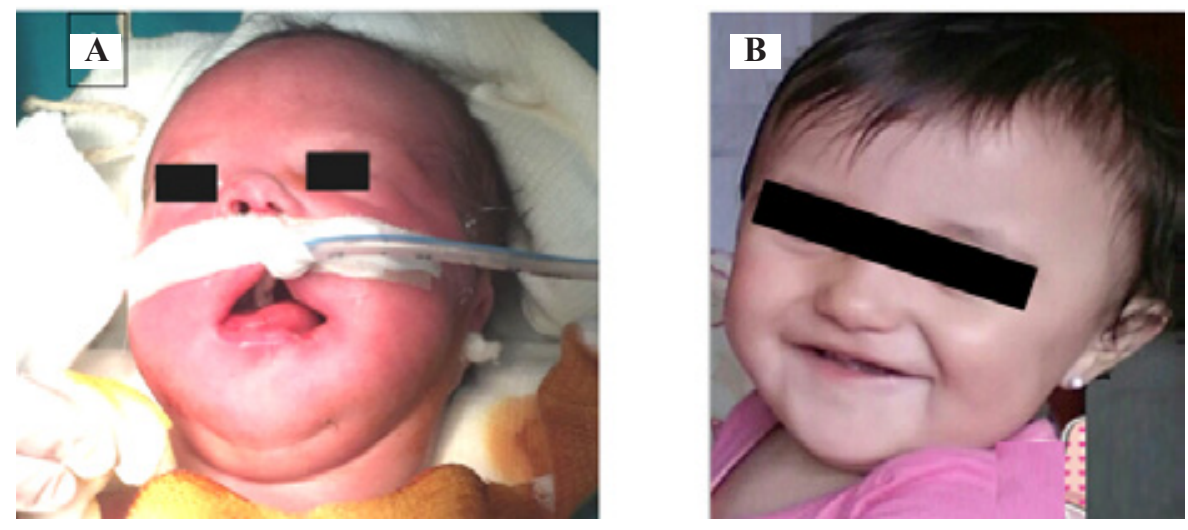

A: paciente en el período posoperatorio inmediato. B: paciente al año de vida. 


\section{DISCUSIÓN}

El teratoma oral o epignathus es una forma poco frecuente de teratoma congénito. El primer caso de este tumor habría sido descrito en $1856 \mathrm{y}$, desde entonces, se publicaron algunas notificaciones. ${ }^{5}$

Si bien hubo varias clasificaciones de acuerdo al lugar de implantación, en la actualidad se utiliza el término epignathus para denominar cualquier teratoma de la cavidad orofaríngea sin especificar el sitio de origen. ${ }^{6}$ Puede asociarse a otras malformaciones congénitas, como anoftalmia, displasia renal bilateral, meningoencefalocele, lengua bífida, síndrome de Pierre Robin e hipoplasia del cuerpo calloso. ${ }^{7}$ Cuando por su gran tamaño dificultan la deglución durante el período fetal, es frecuente que se acompañen de polihidramnios. Se desconoce su etiología, pero los estudios de citogenética han demostrado que el tumor tiene idéntico cariotipo al fetal, lo que apoya al origen mitótico de una célula diploide totipotencial.

La implantación puede ser única o múltiple y la mayoría tiene su punto de fijación en el paladar duro (39\%). Otras localizaciones son: nasofaringe $(34 \%)$, esfenoides $(15 \%)$, orofaringe $(10 \%)$, maxilar $(5 \%)$, etmoides $(5 \%)$ y tabique nasal $(5 \%) .{ }^{8}$ Los casos descritos con extensión intracraneal suelen tener una evolución terminal. ${ }^{9}$

La presentación clínica en el neonato varía según el tamaño de la lesión, puede estar asintomático, o presentar dificultad para alimentarse o dificultad respiratoria. Los epignathus gigantes que llenan la cavidad bucal causan compromiso respiratorio como consecuencia de la compresión de las vías aéreas superiores. Asegurar la vía respiratoria es el mayor desafío que debe enfrentarse en el momento del nacimiento. ${ }^{10}$

El diagnóstico prenatal de los teratomas, mediante las ecografías de control durante la gestación, es fundamental para un tratamiento adecuado y oportuno del recién nacido. El diagnóstico puede dificultarse por la baja frecuencia de aparición de este tumor, su distribución compleja y por su apariencia similar a otras lesiones como quiste dermoide, hamartomas y quiste gastrontestinal. Se puede lograr un diagnóstico prenatal más preciso mediante ecografía tridimensional y RMN, que definen mejor la ubicación y la extensión intracraneal de los tumores, lo que ayuda a disminuir la mortalidad perinatal y la recuperación de los recién nacidos.
La técnica EXIT, descrita por Norris y col. en $1989,{ }^{11}$ consiste en asegurar la vía aérea en forma controlada mediante intubación orotraqueal, fibrobroncoscopia rígida o traqueostomía (de acuerdo con la gravedad de la obstrucción) sin interrumpir la circulación materno-fetal a través del soporte placentario, que ejerce las funciones respiratoria y cardiaca. ${ }^{12} \mathrm{Ha}$ demostrado ser una técnica eficaz para el manejo de fetos con diagnóstico intrauterino de obstrucción de la vía aérea por diferentes causas. ${ }^{13}$ Se debe realizar bajo anestesia general para asegurar la hipotonía del útero y permitir así la adecuada circulación placentaria, disminuyendo el riesgo de prolapso del cordón umbilical y de desprendimiento placentario. ${ }^{14}$

Luego de asegurada la vía aérea del neonato, la TAC y RMN son los estudios por imágenes más útiles. La RMN permite una definición clara de la extensión del tumor y su relación con las estructuras circundantes, mientras que la TAC es particularmente útil para detectar calcificaciones (presentes en el $50 \%$ de los teratomas) y defectos óseos. El contraste vascular permite evaluar la circulación sanguínea de la masa.

La escisión quirúrgica completa seguida de la reparación de las anomalías asociadas, tan pronto como se logre la estabilización del paciente, son el tratamiento de elección.

El pronóstico depende de la extensión y la ubicación de la lesión. El riesgo de una recaída maligna aumenta en caso de la resección incompleta y por la presencia de tejido neural en la lesión. ${ }^{15}$ Por lo general, los teratomas benignos tienen componentes de tejido maduro, aquellos con potencial maligno suelen contener tejido inmaduro. De acuerdo con algunas notificaciones, la recurrencia del teratoma no necesariamente implica su malignidad; sin embargo, se recomienda que los pacientes permanezcan en seguimiento clínico.

En resumen, los teratomas nasofaríngeos congénitos son tumores usualmente benignos, según su extensión y compromiso regional. El diagnóstico prenatal y el aseguramiento de la vía aérea durante el nacimiento son fundamentales para una evolución favorable. La resección quirúrgica completa es considerada el tratamiento definitivo, ya que asegura la permeabilidad de la vía aérea y evita las recurrencias.

\section{REFERENCIAS}

1. Clement K, Chamberlain P, Boyd P, Molyneux A. Prenatal diagnosis of an epignathus: A case report and review of the literature. Ultrasound Obstet Gynecol. 2001;18(2):178-81. 
2. Kumar SY, Shrikrishna U, Shetty J, Sitaram A. Epignathus with fetiform features. J Lab Physicians. 2011; 3(1):56-8.

3. Takagi MM, Bussamra LC, Araujo Júnior E, Drummond $\mathrm{CL}$, et al. Prenatal diagnosis of a large epignathus teratoma using two-dimensional and three-dimensional ultrasound: Correlation with pathological findings. Cleft Palate Craniofac J. 2014; 51(3):350-3.

4. Hames ME, El-Din MH, Abdelazim IA, Shikanova S, et al. Prenatal Diagnosis and Immediate Successful Management of Isolated Fetal Epignathus. J Med Ultrasound. 2019; 27(4):198-201.

5. Escobar GI, Poblete LA, Becker VJ, ZavalaB A, et al. Epignathus: Reporte de un caso y revisión de la literatura. Rev Chil Obstet Ginecol. 2005; 70(3):173-9.

6. Vandenhaute B, Leteurtre E, Lecomte-Houcke M, Pellerin $\mathrm{P}$, et al. Epignathus teratoma: report of three cases with a review of the literature. Cleft Palate Craniofac J. 2000; 37(1):83-91.

7. Bale PM, Reye RD. Epignathus, double pituitary and agenesis of corpus callosum. J Pathol. 1976; 120(3):161-4.

8. Castillo JM, Drut R, Perroni C, Pollono D, et al. Epignatus-
Revisión de casos en el Hospital Pediátrico Sor María Ludovica de La Plata. FASO. 2009; 16(2):1-3.

9. Smith NM, Chambers SE, Billson VR, Laing I, et al. Oral teratoma (epignathus) with intracranial extension: a report of two cases. Prenat Diagn. 1993; 13(10):945-52.

10. Jadhav SS, Korday CS, Malik S, Shah VK, et al. Epignathus Leading to Fatal Airway Obstruction in a Neonate. J Clin Diagn Res. 2017; 11(1):SD04-5.

11. Norris M, Joseph J, Leighton B. Anesthesia for perinatal surgery. Am J Perinatol. 1989; 6(1):39-40.

12. Laje P, Howell LJ, Johnson MP, Hedrick HL, et al. Perinatal management of congenital oropharyngeal tumors: the ex utero intrapartum treatment (EXIT) approach. J Pediatr Surg. 2013; 48(10):2005-10.

13. Masahata K, Soh H, Tachibana K, Sasahara J, et al. Clinical outcomes of ex utero intrapartum treatment for fetal airway obstruction. Pediatr Surg Int. 2019; 35(8):835-43.

14. Hirose S, Farmer DL, Lee H, Nabuhara KK, et al. The Ex Utero Intrapartum Treatment Procedure: Looking Back at the EXIT. J Pediatr Surg. 2004; 39(3):375-80.

15. Hu R, Jiang RS. The recurrence of a soft palate teratoma in a neonate: a case report. Head Neck Oncol. 2013; 5(2):16. 